\title{
ASSESSMENT OF DYNAMIC PARAMETERS OF RAIL FASTENING
}

The paper is devoted to the analysis and comparison of the dynamic parameters of the rail fastening used in a standard way in the core railway network of the Czech Republic, i.e. the elastic rail fastening Vossloh $W 14$ and Pandrol FC. The paper also includes the description of the measurement and evaluation method. The conclusion of the paper is devoted to recommendations for further measurement and practical applications.

Keywords: Rail fastening, signal analysis, impulse excitation, frequency response function.

\section{Introduction}

The constant increase in demands on the load capacity of railway structures leads to greater stiffness of construction layers and subgrade. Concrete sleepers are also almost exclusively used, because have much higher bending stiffness and less flexibility than wooden sleepers. All these factors lead to greater loading on track bed, which changes its shape under increasing load and thereby affects the track geometry parameters. In the ballast track, for the reasons described above, gravel bench behind the heads of sleepers tends to crash. Open spaces between the sleeper and gravel are created, due to the high bending stiffness of concrete sleepers. When a train passes the sleepers rest on the collapsed gravel bench, which creates uneven sleeper support and enhance the dynamic effects, which accelerates the degradation of track bed [1].

To avoid such undesirable phenomena as much as possible, considerable financial resources must be expended to new designs research, as well as to diagnostic quality of track geometry and their potential maintenance [2]. Still, there are some relatively simple measurement procedures, which can well predict the characteristics of the rail fastening and thus also its suitability. The aim of this paper is to point out the use of selected methods of experimental modal analysis to determine the characteristics of rail fastening.

\section{Methods of measurement}

The basic objective of methodology was
comparison of rail fastening test samples in

a laboratory with respect to the vibration response of the results obtained in situ. The method of excitation with impulse hammer was selected. The measured data were analysed using the Frequency Response Function (FRF) [3]. The dynamic responses were measured on the foot rail, on the head of the sleeper and in gravel ballast (except in-situ measurement).

A part of a sleeper with installed fastening system was used as a test sample in the laboratory. The sleeper was placed in gravel bed in shape of a truncated pyramid, which was built in a test box with dimensions of $2.0 \times 2.0 \mathrm{~m}$. The test box was insulated from the base plate by an interlayer of cork.

Furthermore, additional measurements were carried out in situ. For these purposes was selected a test section on line no 260 in the $\mathrm{km} 166.140$ in the area of Bilovice nad Svitavou [4]. For obvious reasons, the exciting with impulse hammer was used in situ too.

\section{Test samples}

In the laboratory, the following types of baseplatesless flexible rail fastening for system UIC 60 and gauge extension + RK $0 \mathrm{~mm}$ were tested [5 and 6]:

1. The part of the sleeper B91P with Fastclip fastening and side insulator 7049

- The fastening Pandrol (flexible clips, FC1501 with insulators 8494, pad under bottom rail 6530)

2. The part of the sleeper B91S/1 with polyamide opened plug

- The fastening system Vossloh W14 (flexible clamps Sk114, angled guides Wfp 14K-12, sleeper screw R1 with pad Uls 7, pad under bottom of the rail WU 7)

\footnotetext{
* 1 Jaroslav Smutny, ${ }^{1}$ Lubos Pazdera, ${ }^{1}$ Vladimir Tomandl, ${ }^{2}$ Herbert Seelmann

${ }^{1}$ Institute of Railway Structures and Constructions, Faculty of Civil Engineering, Brno University of Technology, Brno, Czech Republic

${ }^{2}$ Rail Data Services Austria GmbH \& Co KG, Wien, Austria

E-mail: smutny.j@fce.vutbr.cz
} 
- The fastening system Vossloh W14NT (flexible clamps Skl14, angled guides Wfp 14K NT, sleeper screw R1 with pad Uls 7, pad under bottom of the rail Zw 900 NT)

- The fastening system Vossloh W21NT (flexible clamps Sk121, angled guides Wfp $21 \mathrm{~K}$ NT-12, sleeper screw R1 with pad Uls 7, pad under bottom of the rail $\mathrm{Zw}$ 1000/150 HS-35)

- The fastening system Vossloh E14 (flexible clamps Skl 21, angled guides Wfp 16G/Wfp 16F, sleeper screw R3 with pad Uls 7, pad under bottom of the rail Zw 693/150, steel spread plate Grp 22/150, highly flexible pad Zwp E14 NT/150).

Verifying measurements in situ were performed on fastening Vossloh W14 in track No. 1, or on fastening Pandrol FC I in track No. 2 of the given section of the track. The rail fastening Pandrol FC I and especially Vossloh W14 are the most commonly used without-clip-plate flexible fastenings in the Czech Republic. These can be found in all the upgraded sections of transit railway corridors and tracks of national importance.

Fastening W14NT is equipped with angled guide inserts Wfp 14K NT provided with a special guide rail on the underside. This guide rail in the mounting position enters the area under the track base, avoiding excessive tipping or decline of the track. For this reason, the fastening W14NT is preferably used in track with directional arcs of small radii. Fastening E14 belongs among the fastenings with increased vertical flexibility.

Fastenings E14 is suitable where there is a need to increase the damping of dynamic effects or reduce the emitted noise. It was originally developed for slab track where highly elastic pad Zwp E14 NT/150 replaced the dampening effects of the entire track bed. Currently the trend for fastenings E14 can be observed also in places where it is not possible to establish a sufficient track bed thickness. It is a fastening with a highly elastic underlay under the track base Zw 1000/150 HS-35. This increased flexibility is also consistent with vertical higher fatigue limit of clamps Sk121. Field of application of fastening W21NT is similar to fastening W14NT or E14.

\section{The test arrangement}

Laboratory samples were fitted with rail grid vibration acceleration sensors as shown in Fig. 1. From the perspective of the cross section all sensors were placed in the longitudinal axis of the sleepers. Location of sensors $A r$ and $A s$ was due to the rail web chosen conceptually in accordance with previous measurements [7 and 8]. A special measuring stone was developed at the Institute of Railway Structures and Constructions, due to the location of the sensor $A g b$ to the gravel beneath the mattress platform of sleeper. The $A g b$ sensor was not used in measuring in-situ. The in-situ test arrangement is shown in Fig. 2.

Suitable Bruel\&Kjær accelerometers (type BK 4507B004) were used as sensors. Impact was driven with the impact hammer Bruel\&Kjær, type 8210, with excitation tip hard tip black. Data from measurement were analysed using PULSE 3560D modular analyser from Bruel \& Kjær.

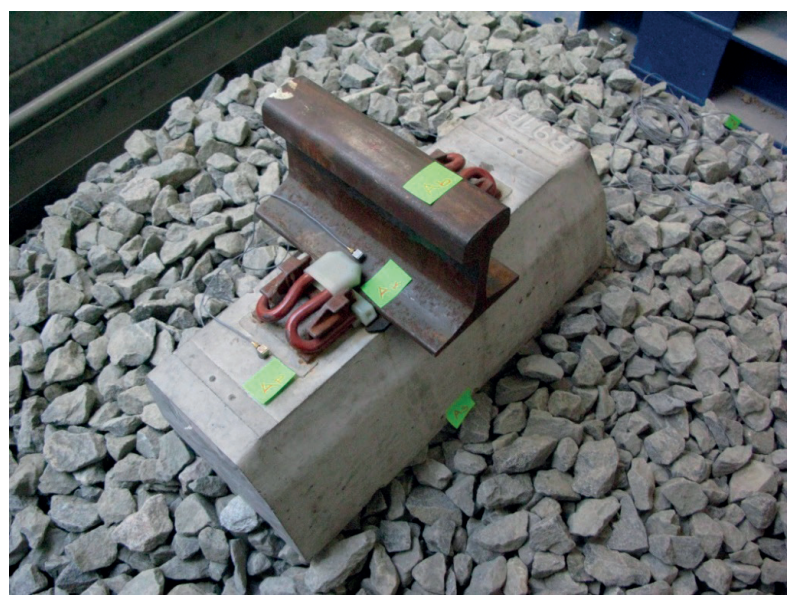

Fig. 1 The test arrangement in-labo, impact excitation

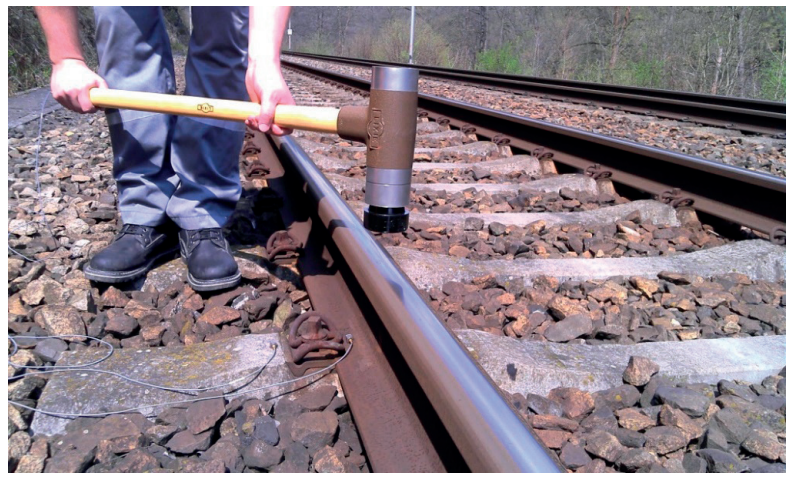

Fig. 2 The test in-situ, impact excitation

\section{Evaluation of the data}

The results are partially shown in Tables $1-2$ and Fig. 3. Assessed value of impulse excitation was the accelerance. Frequency range $1 \div 1000 \mathrm{~Hz}$ was set after the initial testing. In the case of impulse evaluation three repeated laboratory measurements of accelerance were performed for each type of rail fastening, each derived from ten hammer hits. Impulse hammer was used also in the case of the verification in situ. Frequency and survey methodology of accelerance measurement was similar to that in the laboratory. However, in order to guarantee safe 
operation of the track, the influence of the tightening of the propeller was not measured at Vossloh fastening.

The results of individual measurements were averaged and displayed in graphs of frequency response functions H3. Relative damping (damping ratio) of tested structures was determined by half-power bandwidth method. Evaluation was done in software ME'Scope. It is worth noting that the Tables 1 and 2 contain full results for rail fastenings Pandrol FC I and Vossloh W14 only.

The evaluation focused on the low-frequency range of $1 \div 80$ $\mathrm{Hz}$, medium-frequency range $80 \div 400 \mathrm{~Hz}$ and high-frequency range of $400 \div 1000 \mathrm{~Hz}$. This division is based on previous work and is based on the principle that in the low frequency range mainly the effects of the operation of bogies and wheelsets of moving railway vehicles can be observed, middle frequency range is a manifestation of imperfections on the wheel rail contact area, as well as a high frequency range up to $1000 \mathrm{~Hz}$. Here, however, the influence of acoustic phenomena caused by moving vehicles is also manifested.

In the low-frequency range all fastening systems show a significant response between 60 and $80 \mathrm{~Hz}$. The most significant relative damping on the rail $5.3 \%$ is achieved with Vossloh fastening W14NT, the smallest $0.8 \%$ with fastening Vossloh E14. High damping ratio of fastening W14 NT is most likely caused by the guide rail on the bottom surface of the angular guide insert that prevents larger rail movements. On the contrary, the fastening E14 is characterised by an increased vertical flexibility, which may result in increased vibration of the rail. On the sleeper there was the largest structural damping 9.3\% recorded at fastening Vossloh W21NT, the smallest at Pandrol FC I and at Vossloh W14NT (both 3.1\%). The difference between the damping on the rail and on the sleeper can indicate vertical fastening flexibility. Vossloh fastening E14 W and Vossloh W21NT can therefore be defined as the fastening with largest vertical flexibility. The resulting damping $4.3 \pm 2.2 \%$ in the track bed suggests that the densified layer of gravel created stable surface for all tested sets.

There are no significant frequencies for fixing Pandrol FC I on the rail at the medium frequency range, except for frequency 277 $\mathrm{Hz}$ in situ, where the tested structure shows a damping of 3.1\%. This frequency is equivalent to a frequency of $430 \mathrm{~Hz}$, which was recorded in laboratory tests. On the sleeper there are significant frequency ranges of fastening Pandrol frequencies of $145 \div$ $165 \mathrm{~Hz}$, where it reaches an average damping of $5.3 \%$. Greater damping at high frequencies in the track bed was not reached. Neither at Vossloh rail fastening W14 significant frequency was found. This fastening achieves an average damping of $3.7 \%$ on sleeper In this respect, the range of $110 \div 215 \mathrm{~Hz}$ is particularly important. Damping of $6.4 \%$ at frequencies of $115 \div 155 \mathrm{~Hz}$ in gravel was calculated. Fastening W14NT shows damping $4.0 \%$ on the rail, $3.4 \%$ on the sleeper and $4.2 \%$ in the track bed at selected frequencies in the range $110 \div 340 \mathrm{~Hz}$. For fastening Vossloh
W21NT the essential resonance frequency is $290 \mathrm{~Hz}$. Fastening here shows damping of $7.3 \%$ on the rail, $6.3 \%$ on the sleeper and $7.4 \%$ in the gravel. Another frequency spectrum of $130 \div 145 \mathrm{~Hz}$ is not so important; the construction here achieves damping $4.5 \%$. The last tested construction Vossloh E14 shows a high resonant range $120 \div 130 \mathrm{~Hz}$ with damping $3.6 \%$ on the rail, $3.1 \%$ on the sleeper and $3.9 \%$ in the track bed. Even more significant damping of about $5.2 \%$ was obtained at a frequency of $220 \div 265 \mathrm{~Hz}$. Overall, the comparison of the behaviour of structures reveals that with increasing vertical fastening flexibility the size of the resonance range of medium frequencies of 80 to $400 \mathrm{~Hz}$ increases as well. The least favourable in this respect seems to be W21NT Vossloh fastening, fixing Pandrol FC I, or Vossloh fastening E14.

For fastening Pandrol FC I the frequency $430 \mathrm{~Hz}$ is important in the higher frequency range of $400 \div 1000 \mathrm{~Hz}$, where the average damping is $2.6 \%$ and in track bed also frequencies $630 \div 730 \mathrm{~Hz}$ with the calculated damping of $1.9 \%$. For fastening Vossloh W14 damping was found at the large number of high-frequency range. Only the values of range $450 \div 500 \mathrm{~Hz}$ and $600 \div 700 \mathrm{~Hz}$ are, however, higher than $1.0 \%$.

For fastening Vossloh W14NT damping was found in the frequency range $510 \mathrm{~Hz}$ to $600 \mathrm{~Hz}$ in the average of $1.5 \%$.

The relative damping in dependence on the frequency,

Pandrol FC I fastening

Table 1

\begin{tabular}{|c|c|c|c|c|}
\hline \multicolumn{5}{|c|}{ Pandrol FC I fastening } \\
\hline \multirow[t]{2}{*}{ Sensor } & \multicolumn{2}{|c|}{$\begin{array}{c}\text { Laboratory, } \\
\text { Impact excitation }\end{array}$} & \multicolumn{2}{|c|}{$\begin{array}{c}\text { In situ, } \\
\text { Impact excitation }\end{array}$} \\
\hline & $\mathrm{f}[\mathrm{Hz}]$ & Damping [\%] & $\mathrm{f}[\mathrm{Hz}]$ & Damping [\%] \\
\hline \multirow{6}{*}{ 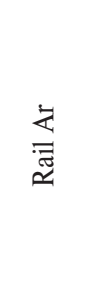 } & 69 & 3.0 & 83 & 3.3 \\
\hline & 167 & 1.3 & 277 & 3.1 \\
\hline & 430 & 3.1 & 359 & 0.4 \\
\hline & 573 & 0.1 & 518 & 0.6 \\
\hline & 650 & 0.1 & 774 & 0.5 \\
\hline & 701 & 0.1 & 931 & 0.1 \\
\hline \multirow{6}{*}{ 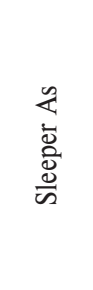 } & 73 & 4.0 & 64 & 2.1 \\
\hline & 114 & 1.7 & 147 & 7.7 \\
\hline & 165 & 2.6 & 278 & 4.6 \\
\hline & 422 & 5.6 & 591 & 0.1 \\
\hline & 651 & 0.1 & 773 & 0.1 \\
\hline & 874 & 0.1 & 798 & 0.1 \\
\hline \multirow{7}{*}{ 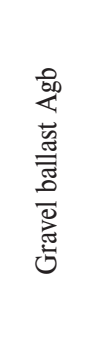 } & 70 & 2.8 & - & - \\
\hline & 89 & 2.8 & - & - \\
\hline & 198 & 2.4 & - & - \\
\hline & 222 & 1.3 & - & - \\
\hline & 437 & 5.3 & - & - \\
\hline & 733 & 4.5 & - & - \\
\hline & 850 & 0.2 & - & - \\
\hline
\end{tabular}


Internal damping of $3.3 \%$ of the corresponding frequencies $410 \div 445 \mathrm{~Hz}$ was also found in track bed. The average damping value of $1.9 \%$ was calculated for Vossloh fastening system W21NT. This damping was achieved at frequencies of $415 \div 445 \mathrm{~Hz}$. The second major frequency is $562 \mathrm{~Hz}$, where the damping of $3.3 \%$ was found. Fastening E14 showed in the selected frequency range $400 \div 1000 \mathrm{~Hz}$ no significant damping values exceeding $0.5 \%$. In the range of higher frequencies all tested fastenings show the diminished ability of vibration damping than in the range below $400 \mathrm{~Hz}$. The least favourable of all tested structures seems to be Vossloh fastening W 14NT that in $510 \mathrm{~Hz}$ to $600 \mathrm{~Hz}$ shows the highest amplitude of the vibration acceleration on the sleeper, hence in the track bed.

From the waveform of frequency response functions it is possible to determine the characteristics indicative for the test of rail fastening in terms of their mounting rigidity and in terms of relative acceleration of the rail towards the sleeper in a vertical direction by quantifying surface between curves $A_{k}$ and $A_{p}$. The presence of the guides on the underside of the guide angle insert fastenings W14NT and W 21NT using low frequency range up to $80 \mathrm{~Hz}$ which causes a very small area between the given curves in this frequency range - relative vertical vibration acceleration transmission from the track to the sleeper is minimal.

In the overall frequency scale, the largest differences between the curves $A_{r}$ and $A_{s}$ are at fastenings E14, the smallest at fastenings W14. Measurement tentatively confirmed the static cutting stiffness of tested sets of rail fastenings listed in company regulations.

Fastening Pandrol FC I shows interesting properties in the resonant frequency range of around $430 \mathrm{~Hz}$. This frequency is a manifestation of corrugation with a period of waves about 7 $\div 9 \mathrm{~cm}$, depending on the driving speed, which is considered in the range typical for corridor route, i.e. 120 to $160 \mathrm{~km} \cdot \mathrm{h}$ ${ }^{-1}$. Using the fastenings Pandrol FC I on routes where there is emergence of corrugation with that wavelength, can accelerate the development of rail defects. Similar characteristics are also shown by fastenings Vossloh W21NT at high frequencies around $290 \mathrm{~Hz}$ or by Vossloh fastenings E14 at frequencies in the range of $220 \div 265 \mathrm{~Hz}$.

At the tested fastenings with maximum static secant stiffness Vossloh W14 and Vossloh W14NT there is the most noticeable shift of resonant range towards higher frequencies. The presence of waves of shorter wavelengths up to $5 \mathrm{~cm}$ can be seen on the corridor lines in the high frequency range. Therefore it follows that in areas where we can expect the development of periodic defects of the rail surface of small and medium-sized lengths it is, due to general properties, suitable to use vertical flexible fastening systems, such as Vossloh E14 or Vossloh W21NT. On the contrary, in places where we can expect rail defects like gliding or long waves, it is preferable to use fastenings with higher static secant stiffness of the rail pad. These include fastening system
The relative damping in dependence on the frequency, Vossloh W14 fastening

Table 2

\begin{tabular}{|c|c|c|c|c|}
\hline \multicolumn{5}{|c|}{ Vossloh W 14 fastening } \\
\hline \multirow{2}{*}{ Sensor } & \multicolumn{2}{|c|}{$\begin{array}{c}\text { Laboratory, } \\
\text { Impact excitation }\end{array}$} & \multicolumn{2}{|c|}{$\begin{array}{c}\text { In situ, } \\
\text { Impact excitation }\end{array}$} \\
\hline & $\mathrm{f}[\mathrm{Hz}]$ & Damping [\%] & $\mathrm{f}[\mathrm{Hz}]$ & Damping [\%] \\
\hline \multirow{7}{*}{ 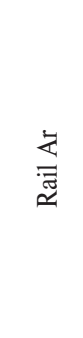 } & 72 & 2.3 & - & - \\
\hline & 128 & 2.3 & 119 & 1.0 \\
\hline & 497 & 0.4 & 595 & 0.6 \\
\hline & 646 & 0.2 & 752 & 0.1 \\
\hline & 772 & 0.3 & 781 & 1.0 \\
\hline & 830 & 0.2 & 802 & 1.8 \\
\hline & 905 & 0.1 & 906 & 0.1 \\
\hline \multirow{9}{*}{ 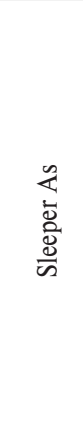 } & 74 & 6.1 & - & - \\
\hline & 96 & 3.2 & 149 & 6.2 \\
\hline & 112 & 3.7 & 305 & 0.3 \\
\hline & 215 & 3.1 & 473 & 0.1 \\
\hline & 499 & 6.9 & 583 & 0.1 \\
\hline & 633 & 0.5 & 792 & 0.1 \\
\hline & 772 & 0.3 & 803 & 0.1 \\
\hline & 831 & 0.2 & - & - \\
\hline & 834 & 0.1 & . & - \\
\hline \multirow{9}{*}{ 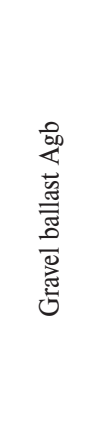 } & 40 & 1.6 & - & - \\
\hline & 72 & 4.5 & - & - \\
\hline & 85 & 2.2 & - & - \\
\hline & 146 & 4.2 & - & - \\
\hline & 222 & 0.3 & - & - \\
\hline & 500 & 0.4 & - & - \\
\hline & 699 & 0.2 & - & - \\
\hline & 751 & 0.1 & - & - \\
\hline & 821 & 0.1 & & \\
\hline
\end{tabular}

Vossloh W14, whether made with a guide rail on the underside of the angular guide insert (type NT) or without.

\section{Conclusion}

Carried out laboratory and operational measurements showed stable performance of all tested types of fastenings for the range of testing. Dependence on the size of the static cutting stiffness and resonant field of fastening systems was revealed. The firmer the fastening the more is the range of the most important frequencies shifted towards higher frequencies.

From the above it can be concluded that, for example, in the Czech Republic operationally validated fastening type Vossloh 

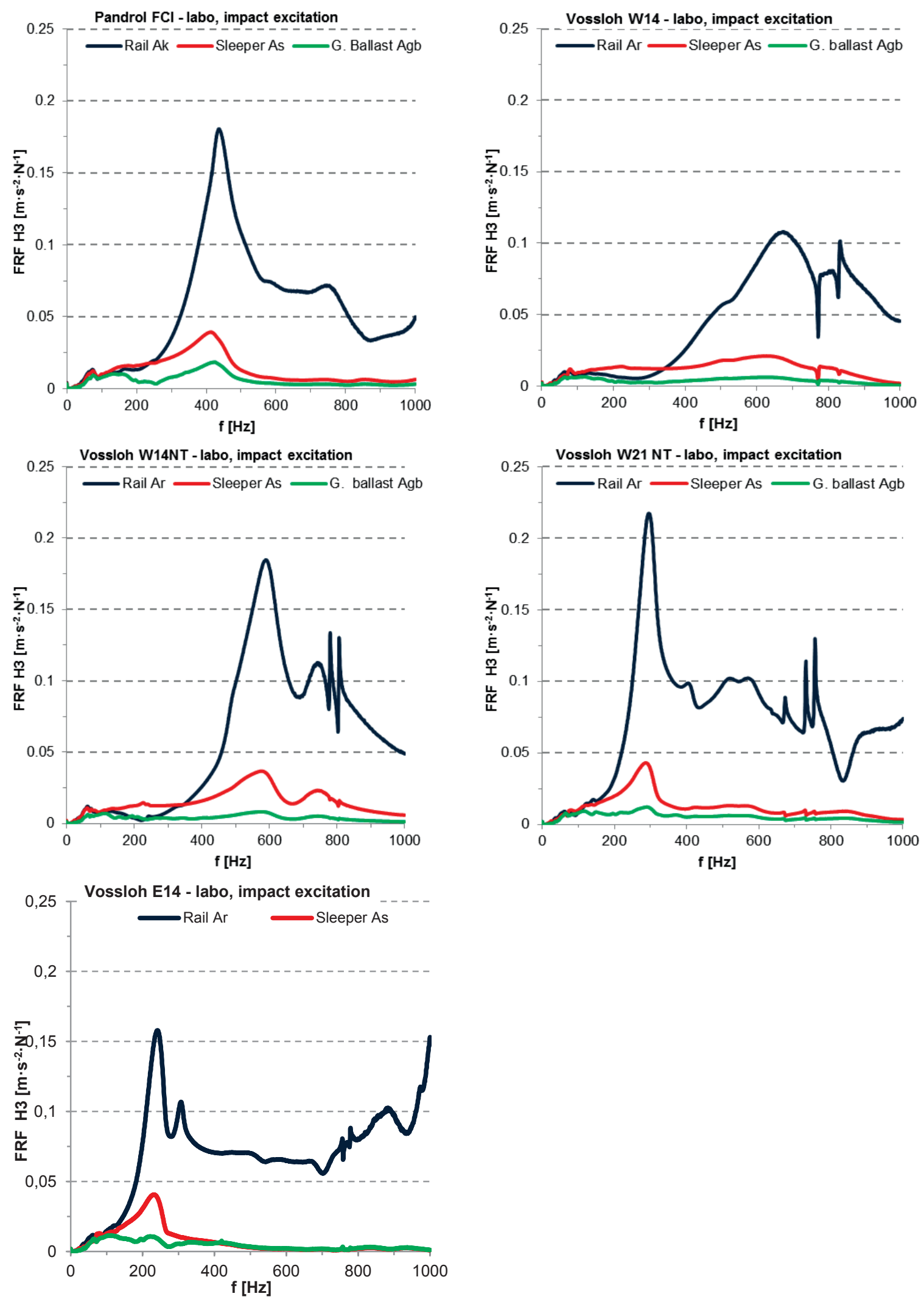

Fig. 3 Frequency characteristics of the testing samples rail fastening, standard tightening torque 
W21NT cannot be recommended to bends with small radii, where there is a risk of emergence of slip waves. These waves are at speeds of 120 to $160 \mathrm{~km} \cdot \mathrm{h}^{-1}$ significant contributors to the level of acceleration in the medium frequency range of $80 \div 400 \mathrm{~Hz}$. For its properties, fastening W21NT could therefore contribute to faster development of the rail defect.

However, based on the data obtained, we can confirm the suitability of each type of tested fastening for the area of application described in the section "test samples". Comparing the results of laboratory measurement with measurements in the field, it could be generally stated that in the in situ measurement a downward trend of significant medium frequency ranges on the rail and on the sleeper of approximately 100 to $150 \mathrm{~Hz}$ toward the beginning was apparent. This was true for both compared the fastenings Pandrol FC I and Vossloh W14.

The cause of this phenomenon has not been shown by measurements. Possible connection is most likely to be seen in the continuously supported rail and the effect of axial forces and stresses in continuous welded rail, which was not taken into account in measurements under laboratory conditions. Slightly different characteristics of both analysed fastenings in higher frequency ranges are then probably also related to the generally complex ties rail - sleeper - rail bed in situ, which cannot be fully simulated under laboratory conditions.

Based upon the analyses made it is possible to say that the used methods offer good results and conclusions. The measured and calculated values prove to be sufficiently accurate and have excellent testing ability.

\section{Acknowledgments}

This paper has been supported by the research project FASTS-15-2806, "The analysis of dynamic response of the railway line structure".

\section{References}

[1] ESVELD, C.: Modern Railway Track, MRT Production: Delft, ISBN 90-800324-3-3, 2001.

[2] SMUTNY, J., PAZDERA, L.: New Techniques in Analysis of Dynamic Parameters Rail Fastening, INSIGHT, ISSN 1354-2575, 2004.

[3] SMUTNY, J., TOMANDL, V., PAZDERA, L.: Dynamic and Acoustic Analysis of Elastic Rail Fastening without Sole Plates, Civil Engineering J., ISSN 1210-4027, Praha, 2009.

[4] SMUTNY, J., PAZDERA, L.: The Experimental Analysis of Dynamic Processes Related to Railway Transport, Monography, ISBN 97880-7204-827-4, Academic publisher CERM: Brno, 2012.

[5] Czech Technical Standards series CSN EN 13481-1 till 5 and CSN EN 13481-7. Railway applications - Track - Performance requirements for fastening systems, 2013.

[6] Czech Technical Standard CSN EN 13146-8. Railway applications - Track - Test methods for fastening systems 2013.

[7] MORAVCIK, M.: Analysis of Vehicle Bogie Effects on Track Structure-Nonstationary Analysis of Dynamic Response, Communications - Scientific Letters of the University of Zilina, ISSN 1335-4205, 2011.

[8] SMUTNY, J., SADLEKOVA, D.: The Vibration Analysis by Margenau-Hill Transformation Method, Communications - Scientific Letters of the University of Zilina, ISSN 1335-4205, 2014. 